\title{
Acute effects of cyclic versus static stretching on shoulder flexibility, strength, and spike speed in volleyball players
}

\author{
Aksel Çelik \\ Department of Physical Education and Sports Teaching, School of Sports Science and Technology, Dokuz Eylul University, İzmir, Turkey \\ Received: February 2016 Accepted: April 2016
}

\begin{abstract}
Objectives: This study aims to examine the acute effects of cyclic stretching versus static stretching on the shoulder flexibility, rotator cuff muscle strength, spike speed, and spike hit rate on target in adolescent volleyball players.

Materials and methods: A total of 36 female volleyball players aged between 13 and 15 years were included in this study. Volleyball players were randomly divided into three groups: cyclic stretching group, static stretching group, and control group. Shoulder flexibility, rotator cuff muscle strength, spike speed, and spike hit rate on target were measured before and after the intervention.

Results: Shoulder flexibility increased in cyclic stretching and static stretching groups after the intervention. Internal rotation strength increased in cyclic stretching group after the stretching intervention, compared to the control group. A significant reduction was observed in the scapular plane abduction strength in static stretching group after the intervention.

Conclusion: Our study results suggest that cyclic stretching may be beneficial, as it increases both the shoulder flexibility and strength of young female volleyball players. In terms of volleyball performance, it can not be declared both cyclic stretching and static stretching have any effect.
\end{abstract}

Keywords: Adolescent; hand dynamometer; shoulder flexibility.

Flexibility and shoulder strength are very important while playing volleyball, particularly in regards to spiking, since the spike is the most explosive movement form of the overhead volleyball skills. It is a complex movement pattern requiring flexibility, muscular strength, coordination and neuromuscular efficiency. ${ }^{[1]}$ The major shoulder motion is the external/internal rotation for volleyball players. ${ }^{[2]}$ Flexibility is defined as the range of motion (ROM) of a joint. Range of motion of the hitting hand may affect the power of the spike. ${ }^{[3,4]}$ Static stretching (SS) is one of the most frequently used methods for the increase of acute flexibility, ${ }^{[5]}$ stretching increases joint ROM.

Further, stretching is applied in different forms: static and cyclic stretching. While, static muscle stretching is the most common form of pre-exercise stretching used in the athletic population, static stretching involves stretching the muscle to the point at which further movement is limited by the muscle's own tension. ${ }^{[3]}$ Although static stretching increases the shoulder flexibility, it also has the risk of inhibiting strength. Since it is applied for a long-term, it can be useful to use different methods to prevent the negative inhibition of SS. Another method is called cyclic stretching, which is practiced in addition to SS. It is a stretching method with short duration and repetition $^{[6-8]}$ Although the importance of warming up before training and matches is undisputable, there are many studies comparing different stretching types using different protocols. ${ }^{[9-11]}$

Although there are many studies related to the acute effect of static stretching on the various performance measurements, there is not a general consensus on the effect, duration and the number of repetitions. ${ }^{[10,12-14]}$ Many studies have shown the relationship between the increase of flexibility and the length of the stretching period. Although it is not certain yet, ${ }^{[15,16]}$ in general,

Corresponding author: Aksel Çelik, PhD. Dokuz Eylül Üniversitesi Spor Bilimleri ve Teknolojisi Yüksekokulu, Beden Eğitimi ve Spor Öğretmenliği Bölümü, 35340 İnciraltı, İzmir, Turkey. e-mail: aksel.celik@deu.edu.tr 
it is advised that static stretches duration should be between 30 and $60 \mathrm{sec}$.

This study found that static stretching decreases strength performance. ${ }^{[17,18]}$ On the other hand, there are several studies that show static stretching does not change strength performance. ${ }^{[10,13,19]}$ Kay et al. ${ }^{[5]}$ reported some results that static stretching had positive effects with regard to duration and the number of repetitions and increases ROM. In literature, there are a limited studies about cyclic stretching duration and the number of repetitions. ${ }^{[6,7,20]}$ Recent studies have provided some evidence that the response of the musculo-articular complex to cyclic and static stretching may be different. ${ }^{[6,20]}$ There are no studies about how the upper extremities were effected by cyclic stretching as compared to static stretching. It can be expected that the stretching method should increase ROM and have a positive effect on the strength without any negative effects.

In recent years, many studies were performed about the lower extremities with few studies about upper extremities ${ }^{[9,21,22]}$ Kay et al. ${ }^{[12]}$ declared that they have clear evidence about lower extremities assuming that a stretch did not affect higher-speed force production when the stretch lasted $45 \mathrm{sec}$.

The hypotheses of this study were; (i) SS would enhance performance regarding flexibility, and spike hit rate on target. In contrast, SS would decrease shoulder strength and spike speed, (ii) - CycS (cyclic stretching) would also enhance performance regarding flexibility, and spike hit rate on the target, in addition $\mathrm{CycS}$ would enhance or have neutral effects on both shoulder strength and spike speed.

\section{MATERIALS AND METHODS}

This study used a randomized-controlled trial design with repeated measures. We evaluated the effects of two different stretching protocols on shoulder flexibility. Before the experimental procedures were undertaken, each volleyball player visited the sport center to receive instruction about the study and to participate in a familiarization trial to practice back scratch test, spike skill assessment ${ }^{[23]}$ where the spike speed data of the participants were recorded in another session to be used for the reliability statistics followed by the rotator cuff muscle strength test ${ }^{[24,25]}$ on a volleyball court. These familiarization trials were preceded by CycS and SS protocols. The participants were randomly divided into three groups: cyclic stretching group (CycSG), static stretching group (SSG) and control group (CG).

The back scratch test was performed to assess the shoulder flexibility level using a measuring tape. Upper extremity length was measured from acromion to ulnar styloid. The rotator cuff muscle strength was measured via a hand dynamometer.

Spike hit rate on the target and the spike speed were calculated during the assessment of spike skills test. Dominant arms of volleyball players were used for spike skills and all other evaluations. Measurements were applied before (pre) and after (post) stretching assessment. Volleyball players from each stretching group practiced both tests randomly over two days. We started the first day with the measurements of the strength assessments. Firstly, back scratch test and strength test were measured. After these pre-tests, volleyball players practiced $\mathrm{CycS}$ and SS methods. After stretching practices, back scratch test and strength test were applied successively. On the second testing day measurements related to spike skill assessments were performed. Here we started with the back scratch test. Afterwards, all participants tried to spike the ball at the target. CycSG and SSG practiced stretching according to previously determined stretching procedures. The back scratch test and spike skill assessments were applied successively. The evaluations took nearly $30 \mathrm{~min}$. All data was collected, at the same time of day: between 15:00-17:00.

\section{Study participants}

Thirty-six young female volleyball players aged between 13 and 15 years voluntarily participated in this study. All participants were free from musculoskeletal injuries, and able to perform stretching protocols. All participants abstained from vigorous physical activity 48 hours before each experimental session. This study was approved by the Dokuz Eylül University Research Ethics Committee (protocol approval number 2015/15-2). Since all participants were younger than 18 years old, the parents or legal guardians provided the participation approval. The study was conducted in accordance with the principles of the Declaration of Helsinki.

\section{Procedures}

The participants were randomly divided into three equal groups: CycSG, SSG and CG. CycSG performed two sets of three 15 -sec stretches $(2 \times 3 \times 15 \mathrm{sec})$ with a 10 -sec rest between repetitions and a 15 -sec rest between sets. Static stretching group performed two sets of $45-\mathrm{sec}$ stretches $(2 \times 45 \mathrm{sec})$ with a $15-\mathrm{sec}$ 
rest between sets. Control group performed no stretching exercises (sitting still for $90 \mathrm{sec}$ ). The participants did not warm up prior to the stretching assessment. Stretching was performed actively by the volleyball players. CycS and SS consisted of slow active movement (maneuver) of shoulder for internal rotation. A summary of experimental and testing procedures are shown in Figure 1.

During CycS and SS tests the dominant arm was at the internal rotation and the dorsal side of the hand was at the back. Every stretching trial was applied according to the farthest point that could be reached in at least two sec. The participants reported a feeling of maximal stretch without pain.

\section{Shoulder flexibility}

In the back scratch test; the dominant arm was measured via a tape measure during both pre-test and posttest. The distance measured between the middle fingers of the hands is the test score. If the fingers were overlapped, the value is positive "+", otherwise, it was negative "-". The best two successive trials were considered. Results were recorded with an accuracy of $0.5 \mathrm{~cm} .{ }^{[26]}$

\section{Strength tests}

Rotator cuff muscle strength was measured with a Powertrack II (JTech, New York, ABD) hand dynamometer. The mean of the three successive tests were calculated. All participants were measured in a sitting position with knees at $90^{\circ}$ flexion, sitting upright. External rotation strength was tested while elbow is at $90^{\circ}$ flexion, $0^{\circ}$ abduction, at the direction from wrist forming external rotation (Figure 2-A1). Internal rotation strength is tested while elbow is at $90^{\circ}$ flexion, $0^{\circ}$ abduction, at the direction from wrist forming internal rotation (Figure 2-A2). Scapular plane abduction strength is tested while the shoulder is at $90^{\circ}$ elevation, external rotation and elbow extension, resistance is tested from the wrist in a downward direction (Figure $2 b$ )..$^{[25,27]}$

\section{Spike skill assessment}

All participants hit spike five times and the mean values was used for analysis. ${ }^{[28]}$ The volleyball players were oriented to hit the diagonal spike as fast as possible towards the $16 \mathrm{~m}^{2}(4 \mathrm{x} 4 \mathrm{~m})$ target. Spike direction is limited to this target. The participant hit spike with the volleyball ball from position 4 to position 5. The ball used for the test was a FIVB official match ball. The net height was $2.10 \mathrm{~m}$ during all measurements. A radar gun was used to measure the speed of the ball (Stalker Sport 2, Applied Concepts, Inc. Texas, USA). ${ }^{[29]}$ A radar gun was placed $50 \mathrm{~cm}$ behind the opposite corner of the court in a diagonal position (Figure 3 ).

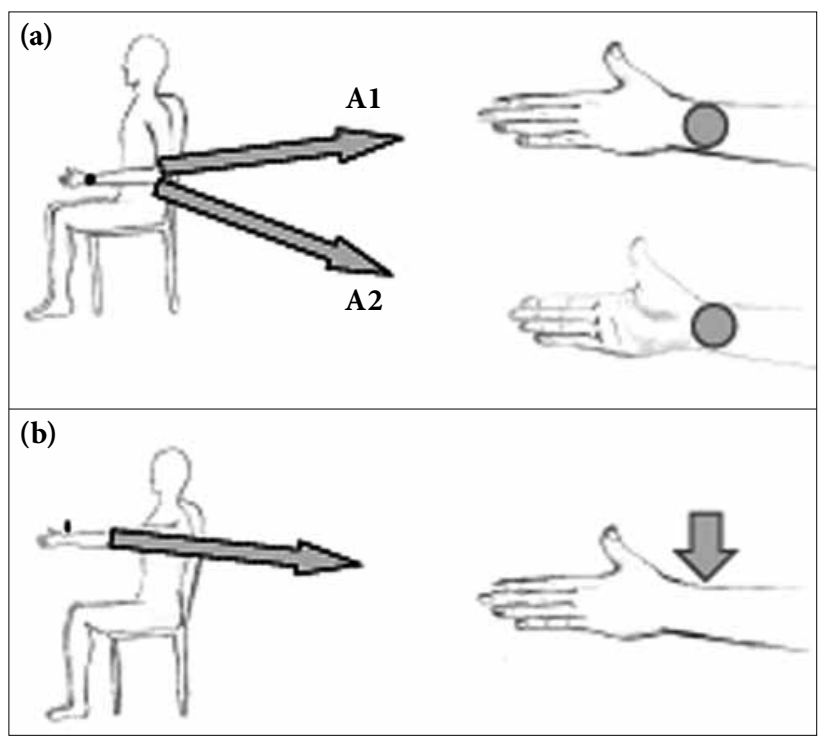

Figure 2. Strength test. (a) A1: Participant positioned on a chair for external rotation strength test (A1) and internal rotation (A2) (A1 and A2: circles indicate the arm location where perpendicular force was applied). (b) Participant positioned on a chair for scapular plane abduction strength test (b: vertical arrow indicates the direction of applied force)

Figure 1. A summary of the experimental method. 


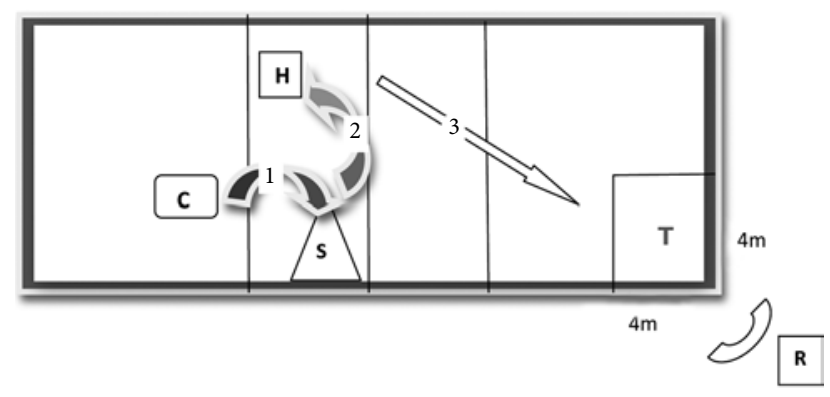

Figure 3. Spike skill assessment test. Here, the ball was thrown by the coach to the setter. All participants hit to the diagonal spike towards the $16 \mathrm{~m}^{2}(4 \times 4 \mathrm{~m})$ target. C: Coach; H: Hitter; S: Setter; T: Target; R: Radar (The numbers indicate the sequence of ball movement.

\section{Statistical analyses}

The statistical analyses were performed using IBM SPSS version 22.0 software (IBM Corp., Armonk, NY, USA). Shapiro-Wilk test was performed to check for normality and assumption of homogeneity of variances was tested using Levene's test. Descriptive statistics were reported either by mean \pm standard deviation or median [minimum - maximum] values depending on the type of performed statistical analyses tests (parametric or non-parametric test). 3x2 (group $\mathrm{x}$ time) mixed design analyses of variance (ANOVA) were used to investigate the interaction effect of time and intervention on dependent performance variables. Then, the effects of intervention/control on performance measures were investigated using paired Student's t-test within each group. Wilcoxon signed-rank test was used where parametric test assumptions were violated. Possible differences in performance changes $(\Delta)$ after interventions between study groups were checked using one way ANOVA with post-hoc Tukey's test. Kruskal-Wallis test together with Mann-Whitney U test was used in the case of violation of parametric test assumptions. Baseline performance measures and reliability levels were evaluated according to intraclass correlation coefficient (ICC) with
95\% confidence intervals. A $p$ value of $\leq 0.05$ was considered statistically significant.

\section{RESULTS}

Age, height, mass, body mass index (BMI), upper extremity length did not differ between study groups. ( $>0.05)$ (Table 1).

The test-retest ICCs (test reproducibility results) for the stretching test (the results of the first and the second days measurements were evaluated) and spike speed (the results of the familiarization and the first days measurements were evaluated) were 0.78 (0.62-0.88) and 0.92 (0.87-0.95), respectively. These values were classified as being in strong agreement. CG did not demonstrate any statistically significant change in any of the dependent variables both in strength assessment day (Table 2) and spike skill assessment days (Table 3) (no time effect).

Mixed design ANOVA results revealed that there was a significant group $\times$ time interaction effect for internal rotation strength $(F[2,33]=3.33, p=0.048$, $\left.\eta \mathrm{p}^{2}=0.168\right)$ indicating that the performance changes were not parallel within the study groups. In contrast, no significant interaction effect was detected for external rotation strength $(\mathrm{F}[2,33]=0.377, \mathrm{p}=0.689$, $\left.\eta p^{2}=0.022\right)$ and spike speed $(\mathrm{F}[2,33]=1.43, \mathrm{p}=0.253$, $\eta \mathrm{p}^{2}=0.080$ ) (Table 2).

There was a significant change in back scratch test between the pre-test and the post-test on strength assessment day in both CycSG and SSG ( $\mathrm{p}=0.002$ and $\mathrm{p}=0.009$, respectively). Also, a significant change in scapular plane abduction strength between pre-test and post-test on strength assessment day in SSG $(p=0.012)$ was observed in addition to a significant difference in internal rotation strength change $(\Delta)$ between CycSG and CG ( $\mathrm{p}=0.038)$ (Table 2).

None of the study groups showed statistically significant change in internal, external rotation strength after interventions $(p>0.05)$. There was

Table 1. Descriptive statistics of participants

\begin{tabular}{|c|c|c|c|c|c|c|c|c|c|c|}
\hline & \multicolumn{3}{|c|}{ CycSG $(n=12)$} & \multicolumn{3}{|c|}{ CG $(n=12)$} & \multicolumn{3}{|c|}{ SSG $(n=12)$} & \multirow[b]{2}{*}{$p$} \\
\hline & Mean \pm SD & Median & Min-Max & Mean \pm SD & Median & Min-Max & Mean \pm SD & Median & Min-Max & \\
\hline Age (year) & $14.0 \pm 0.9$ & & & $13.8 \pm 0.8$ & & & $13.8 \pm 1.6$ & & & ${ }^{\mathrm{A}} 0.26$ \\
\hline Height $(\mathrm{cm})$ & $169.0 \pm 9.2$ & & & $170.0 \pm 10.1$ & & & $168.0 \pm 9.4$ & & & ${ }^{\mathrm{A}} 0.14$ \\
\hline Weight (kg) & & 57 & $48-68$ & & 56 & $47-66$ & & 58 & $49-69$ & ${ }^{\mathrm{K}} 0.17$ \\
\hline $\operatorname{BMI}\left(\mathrm{kg} / \mathrm{m}^{2}\right)$ & $21.5 \pm 2.2$ & & & $20.6 \pm 1.9$ & & & $22.8 \pm 2.1$ & & & ${ }^{\mathrm{A}} 0.25$ \\
\hline Arm length $(\mathrm{cm})$ & $53.9 \pm 3.1$ & & & $55.5 \pm 3.1$ & & & $54.3 \pm 2.7$ & & & ${ }^{\mathrm{A}} 0.39$ \\
\hline
\end{tabular}

CycSG: Cyclic stretching group; CG: Control group; SSG: Static stretching group; Min: Minimum; Max: Maximum; p value of ANOVA or Kruskal Wallis; SD: Standard deviation; BMI: Body mass index; ${ }^{A}$ ANOVA [p value]; ${ }^{\mathrm{K}}$ Kruskal Wallis. 


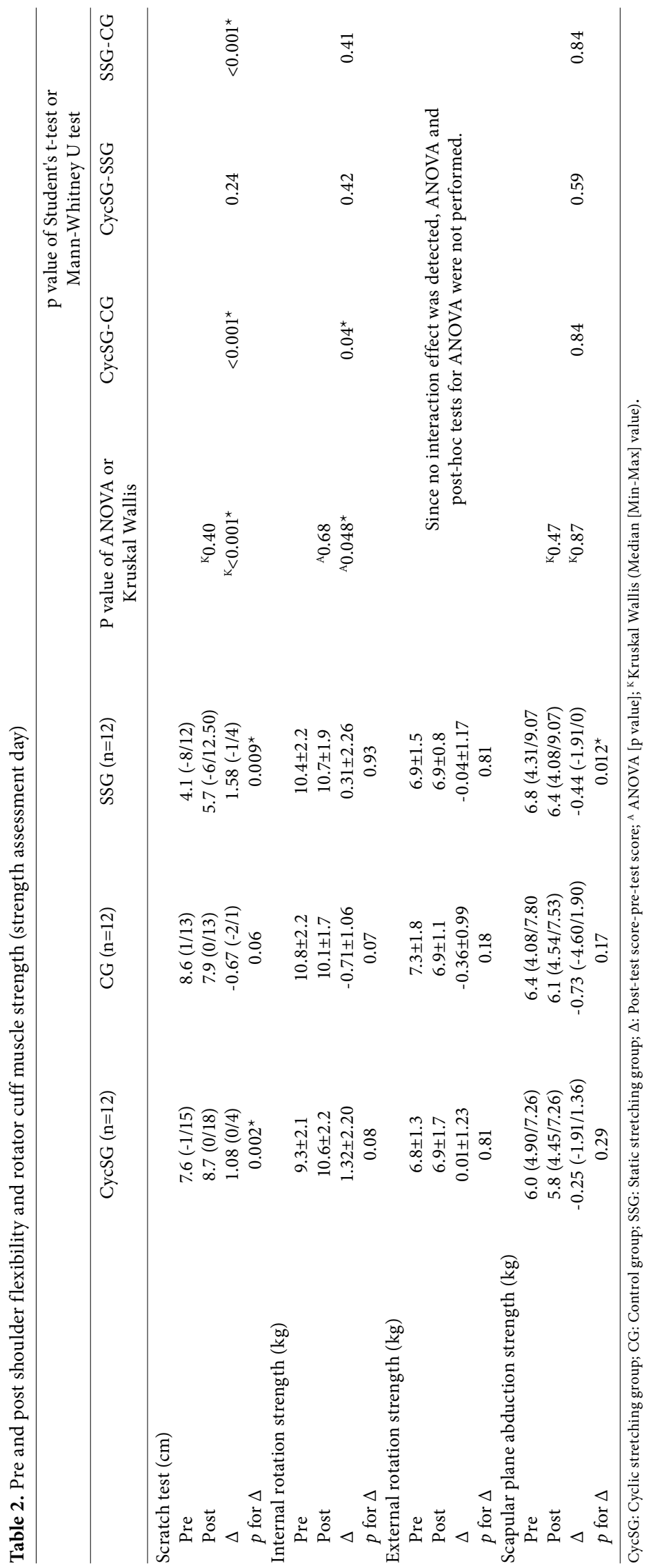




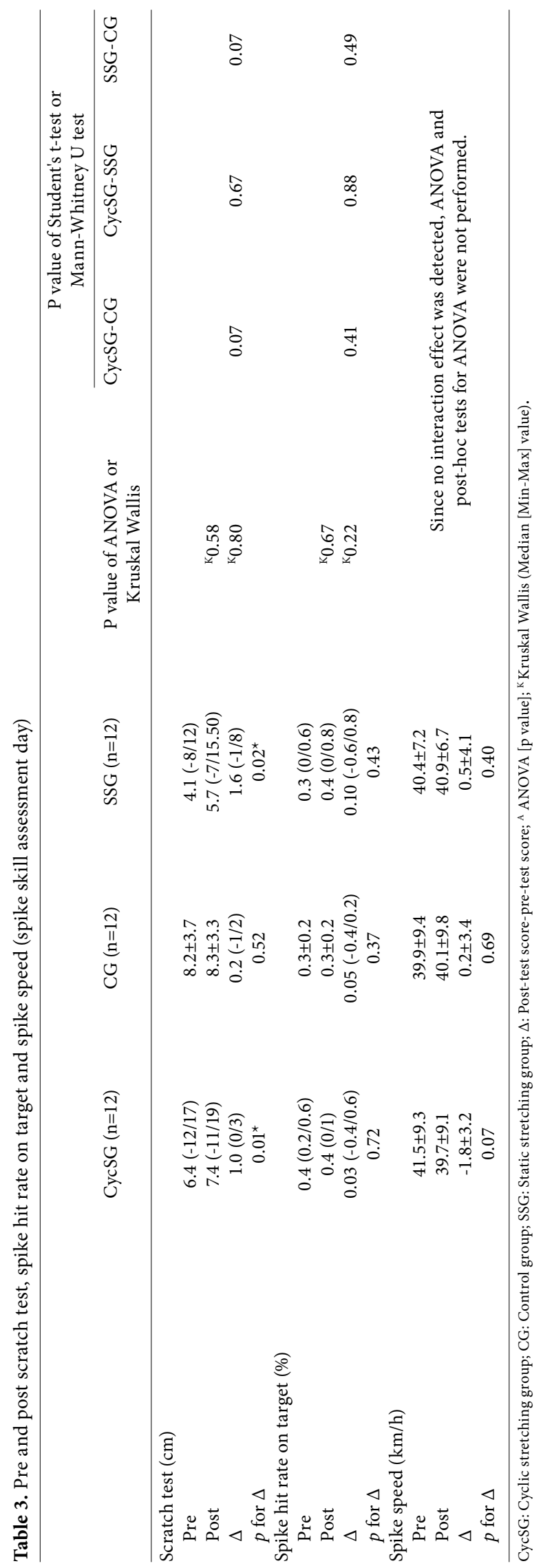

no significant change in scapular plane abduction strength between pre-test and post-test on strength assessment day in CycSG ( $\mathrm{p}>0.05)$. Also, no significant difference in external rotation strength change $(\Delta)$ between study groups ( $p>0.05)$ occurred in addition to an absence of significant change between pre-test and post-test, both on strength and spike skill assessment days ( $>0.05$ ) (Table 2).

There was a significant change to a similar extent in back scratch test after intervention on spike skill assessment day both in CycSG $(\mathrm{p}=0.010)$ and SSG $(\mathrm{p}=0.022)$. There was no significant change after interventions in spike speed and spike hit rate on the target in any of the study groups ( $\mathrm{p}>0.05)$ (Table 3$)$.

\section{DISCUSSION}

Our study is the first one to specifically examine the acute effects of $\mathrm{CycS}$ and SS interventions on strength level (internal rotation strength, external rotation strength and scapular plane abduction strength) and spike skill level (spike speed, spike hit rate on target).

The results revealed that both SS and CycS protocol applied in this study were effective to increase shoulder flexibility. Scapular plane abduction strength decreased after SS intervention. As described previously, SS reduces muscular performance for instance; strength and power production of the stretched muscle group. ${ }^{[1,30,31]}$ Present study results that are supported by previous findings, ${ }^{[21,28]}$ suggest that CycS and SS did not significantly affect rotator cuff muscles and spike speed and spike hit on the target.

The main finding of this study supported our hypothesis that $90 \mathrm{sec}(2 \mathrm{set}$ of $3 \times 15 \mathrm{~s})$ CycS intervention increased internal rotation strength. This data support the hypothesis of enhancing or having no negative or neutral effects on the shoulder strength following CycS.

Gonzalez et al. ${ }^{[32]}$ used a stretching protocol similar to that which was used in CycSG and reported an improvement in performance after stretching exercise for jump performance (three stretches for $15 \mathrm{sec}$ ). CycSG stretching procedure implementation produced similar characteristics with Gonzalez's findings. According to this study's results, it can be declared that CycS has similar effects both on lower and upper limb. ${ }^{[20]}$

The decrease in scapular plane abduction strength after $90 \mathrm{sec}$ (2 set of $45 \mathrm{~s}$ ) SS intervention supported the previous results in literature ${ }^{[8,14]}$ and provided evidence that the decrease following SS 
could be explained primarily by the acute increases in muscle resting length. ${ }^{[3,6]}$ Several studies showed that SS which is longer than $60 \mathrm{~s}$ decreases strength performance ${ }^{[17,18]}$ Kay et al ${ }^{[5]}$ declared that they have clear evidence for lower extremity that static stretch did not affect higher-speed force production if the stretch duration was $<45$-sec. It cannot be denied that stretching duration is as important as stretching type in improving volleyball player's performance.

However, several studies have reported no differences in strength and power performance following SS. ${ }^{[19,21,33]}$ The reason why SS does not change internal and external strength can be its short-term effect on the viscoelastic properties of the rotator cuff muscle group. It is still not absolutely certain whether it enhances muscle strength according to the stretching duration.

Magnusson et al. ${ }^{[34]}$ showed that three sets of $45 \mathrm{sec}$ stretches did not affect the hamstring muscle in the external strength and scapular plane abduction strength. CycS did not manifest the expected stretching effect on these muscles in terms of assessment process and repetition. Here, planning and implementation of further studies including the different duration and number of repetitions will enable us to have clearer evidence.

In the study about the effects of different stretching methods (static, dynamic and combined stretching) on upper body muscular performance, Torres et al. ${ }^{[22]}$ reported that different stretching methods do not change performance. Mascarin et al. ${ }^{[28]}$ reported that the total speed during the handball throwing test was not influenced by SS. Other researchers found that in upper body muscles, SS does not have any effect on serve speed. ${ }^{[35,36]}$ Similarity this study and previous studies showed that CycS and SS did not affect volleyball players spike speed. In contrast to our hypothesis, the reason why it does not change is related to the fact that the spike is a complex movement pattern requiring flexibility, muscular strength, coordination and neuromuscular efficiency.

Cohen et al. ${ }^{[37]}$ reported that it could be possible for a tennis player to increase serve velocity by means of specifically directed muscular strengthening or stretching regimens. Results of Cohen's study revealed that strength and flexibility are related to serve velocity. Sufficiently large ROM before hitting the ball is the main factor of spiking technique. ${ }^{[4]}$ Active stretching exercises are commonly performed in sports and the mechanisms that suggested such changes are not well-known.
It is known that the strength performance of the dominant shoulder (internal rotators) is correlated with spike velocity. ${ }^{[30,38]}$ However strength and spike speed may be connected and it cannot be said that strength affects the performance in volleyball skill. Performing the movements, including SS, before strength training is not suitable, as shown by this study.

Nordez et al ${ }^{[6]}$ reported that the musculo-articular complex response to CycS and SS may be different. Different mechanisms can be effective depending upon the type of stretching procedure performed and analyzing the stretching and muscular strengthening physiological mechanisms according to the skill level of volleyball players will give more detailed information. ${ }^{[9,30]}$

Several studies showed that static stretching increased flexibility. ${ }^{[15,16,39]}$ Even if there are limited studies on cyclic stretching, it has been pointed out that it causes ROM rise for lower limbs. ${ }^{[7]}$ These findings ${ }^{[4,7]}$ support the hypothesis of shoulder flexibility increase in both CycSG and SSG. According to these results, it is considered that the effects of cyclic and static stretching are similar. It has been suggested that static and cyclic stretching increased ROM by increasing the stretch tolerance while the viscoelastic characteristics of the muscle remain unaltered. ${ }^{[40]}$ There are two main ideas (viscoelastic effects and neural effects) to describe the acute effects of stretching ${ }^{[28]}$ Both these effects implications, but the mechanism for the stretching in performance remains unknown. Since few studies have focused primarily upon the upper limbs, many more studies are needed to clarify whether stretching influences performance or not.

Despite the increase in shoulder flexibility, there is no change in both CycSG and SSG for spike hit rate on target. Actually, the increase of shoulder flexibility in elite volleyball players affects performance positively, ${ }^{[4]}$ because the studied group is at young age and spike is a close-kinetic chain skill. In addition, these results show that there is a strong connection between maximal isometric strength production and explosive strength performance changes produced in a short period of time.

Nonetheless, this study has some limitations. Firstly, each group consists of 12 volleyball players. Additionally, spike speed and spike hit rate on target are not the only factors determining spike performance. Another study limitation was the young age of the volleyball players because elite volleyball players may have increased the probability of finding 
statistical significances, particularly spike hit rate on the target. Our aim was to analyze the effects of acute CycS and SS on the rotator cuff muscle, so the leg muscles were not stretched. In addition, Knudson et al. ${ }^{[35]}$ showed in his results that the SS of the upper body did not have any effect on tennis serve velocity and accuracy.

In conclusion, both $\mathrm{CycS}$ and SS interventions were effective in improving shoulder flexibility. Static stretching increased shoulder flexibility but decreased scapular plane abduction strength. CycS is likely beneficial because it increases both shoulder flexibility and strength in young female volleyball players. In general, one of the aspects that needs to be clarified is the acute effects of CycS related to strength and spike speed. Overall, more finding are necessary to determine the optimal stretching duration as well as stretching method to be chosen for upper extremity analysis.

CycS and SS can be used interchangeably to increase the flexibility of young female volleyball players, but during isometric strength training using CycS instead of traditional stretching exercises will be more suitable since it does not cause strength inhibition. Both, SS and $\mathrm{CycS}$ application is considered neutral on the volleyball performance.

\section{Acknowledgements}

I thank Ekim Pekünlü ( $\mathrm{PhD}$ ) for his contributions to the statistical analyses and the drafting of the manuscript. I would also like to thank Sevgi Sevi Yeşilyaprak ( $\mathrm{PhD}$, Assist. Prof.) for her valuable feedback on the drafts of this article and her contribution to the muscle strength assessment. I would like to thank Şule Düzdemir and Harun Muayad Said (Ph.D, Assist. Prof.) for reviewing the English version of the manuscript.

\section{Declaration of conflicting interests}

The author declared no conflicts of interest with respect to the authorship and/or publication of this article.

\section{Funding}

The author received no financial support for the research and/or authorship of this article.

\section{REFERENCES}

1. Wilk KE, Meister K, Fleisig G, Andrews JR. Biomechanics of the overhead throwing motion. Sports Med Arthrosc 2000;8:124-34.

2. Dillman CJ, Fleisig GS, Andrews JR. Biomechanics of pitching with emphasis upon shoulder kinematics. J Orthop Sports Phys Ther 1993;18:402-8.

3. Alter MJ. Science of Flexibility. 3rd ed. Champaign: Human Kinetics; 2004.
4. Liu LF, Liu GC, Sue CW, Huang CF, editors. The application of range of motion (rom) and coordination on volleyball spike. ISBS Conference July 14-18, 2008; Seoul, Korea.

5. Kay AD, Blazevich AJ. Moderate-duration static stretch reduces active and passive plantar flexor moment but not Achilles tendon stiffness or active muscle length. J Appl Physiol (1985) 2009;106:1249-56.

6. Nordez A, McNair PJ, Casari P, Cornu C. Static and cyclic stretching: their different effects on the passive torqueangle curve. J Sci Med Sport 2010;13:156-60.

7. Starring DT, Gossman MR, Nicholson GG Jr, Lemons J. Comparison of cyclic and sustained passive stretching using a mechanical device to increase resting length of hamstring muscles. Phys Ther 1988;68:314-20.

8. Avela J, Kyröläinen H, Komi PV. Altered reflex sensitivity after repeated and prolonged passive muscle stretching. J Appl Physiol (1985) 1999;86:1283-91.

9. Leone DC, Pezarat P, Valamatos MJ, Fernandes O, Freitas S, Moraes AC. Upper body force production after a low-volume static and dynamic stretching. Eur J Sport Sci 2014;14:69-75.

10. Ayala F, De Ste Croix M, Sainz de Baranda P, Santonja F. Acute effects of static and dynamic stretching on hamstrings' response times. J Sports Sci 2014;32:817-25.

11. Kovacs M. Dynamic stretching. The revolutionary new warm-up method to improve power, performance and range of motion. Canada: Ulysses Press; 2009.

12. Kay AD, Blazevich AJ. Effect of acute static stretch on maximal muscle performance: a systematic review. Med Sci Sports Exerc 2012;44:154-64.

13. Cipriani DJ, Terry ME, Haines MA, Tabibnia AP, Lyssanova $O$. Effect of stretch frequency and sex on the rate of gain and rate of loss in muscle flexibility during a hamstringstretching program: a randomized single-blind longitudinal study. J Strength Cond Res 2012;26:2119-29.

14. Kokkonen J, Nelson AG, Cornwell A. Acute muscle stretching inhibits maximal strength performance. Res Q Exerc Sport 1998;69:411-5.

15. Cipriani D, Abel B, Pirrwitz D. A comparison of two stretching protocols on hip range of motion: implications for total daily stretch duration. J Strength Cond Res 2003;17:274-8.

16. Rubley MD, Brucker JB, Knight KL, Ricard MD, Draper DO. Flexibility retention 3 weeks after a 5-day training regime. Journal of Sport Rehabilitation 2001;10:105-12.

17. Feland JB, Myrer JW, Schulthies SS, Fellingham GW, Measom GW. The effect of duration of stretching of the hamstring muscle group for increasing range of motion in people aged 65 years or older. Phys Ther 2001;81:1110-7.

18. Ferguson SL, Kim E, Seo DI, Bemben MG. Comparing the effects of 3 weeks of upper-body vibration training, vibration and stretching, and stretching alone on shoulder flexibility in college-aged men. J Strength Cond Res 2013;27:3329-34.

19. Cramer JT, Housh TJ, Johnson GO, Miller JM, Coburn JW, Beck TW. Acute effects of static stretching on peak torque in women. J Strength Cond Res 2004;18:236-41.

20. Nordez A, McNair P, Casari P, Cornu C. Acute changes in hamstrings musculo-articular dissipative properties induced by cyclic and static stretching. Int J Sports Med 2008;29:414-8. 
21. Haag SJ, Wright GA, Gillette CM, Greany JF. Effects of acute static stretching of the throwing shoulder on pitching performance of national collegiate athletic association division III baseball players. J Strength Cond Res 2010;24:452-7.

22. Torres EM, Kraemer WJ, Vingren JL, Volek JS, Hatfield DL, Spiering BA, et al. Effects of stretching on upper-body muscular performance. J Strength Cond Res 2008;22:1279-85.

23. Rhoads MC. Learning to spike in volleyball with verbal and visually-enhanced feedback. University of Northern Colorado; 2012.

24. Hurd WJ, Kaplan KM, ElAttrache NS, Jobe FW, Morrey BF, Kaufman KR. A profile of glenohumeral internal and external rotation motion in the uninjured high school baseball pitcher, part II: strength. J Athl Train 2011;46:289-95.

25. Michener LA, Boardman ND, Pidcoe PE, Frith AM. Scapular muscle tests in subjects with shoulder pain and functional loss: reliability and construct validity. Phys Ther 2005;85:1128-38.

26. Jones CJ, Rikli RE. Measuring functional fitness of older adults. The Journal on Active Aging 2002; March April: 24-30.

27. Reinold MM, Wilk KE, Fleisig GS, Zheng N, Barrentine SW, Chmielewski T, et al. Electromyographic analysis of the rotator cuff and deltoid musculature during common shoulder external rotation exercises. J Orthop Sports Phys Ther 2004;34:385-94.

28. Mascarin NC, Vancini RL, Lira CA, Andrade MS. StretchInduced Reductions in Throwing Performance Are Attenuated by Warm-up Before Exercise. J Strength Cond Res 2015;29:1393-8.

29. Hikita Y. The acute effects of kinesio taping on throwing velocity in NCAA Division I, II, and III baseball pitchers 2009. Available from: http://libweb.calu.edu/thesis/Hikita_ cup_6020M_10006.pdf

30. Fowles JR, Sale DG, MacDougall JD. Reduced strength after passive stretch of the human plantarflexors. J Appl Physiol (1985) 2000;89:1179-88.

31. Nelson RT, Bandy WD. An update on flexibility. Strength \& Conditioning Journal 2005;27:10-6.

32. González-Ravé JM, Machado L, Navarro-Valdivielso F, Vilas-BoasJP. Acute effects of heavy-load exercises, stretching exercises, and heavy-load plus stretching exercises on squat jump and countermovement jump performance. J Strength Cond Res 2009;23:472-9.

33. Young W, Elliott S. Acute effects of static stretching, proprioceptive neuromuscular facilitation stretching, and maximum voluntary contractions on explosive force production and jumping performance. Res Q Exerc Sport 2001;72:273-9.

34. Magnusson SP, Aagaard P, Nielson JJ. Passive energy return after repeated stretches of the hamstring muscle-tendon unit. Med Sci Sports Exerc 2000;32:1160-4.

35. Knudson DV, Noffal GJ, Bahamonde RE, Bauer JA, Blackwell JR. Stretching has no effect on tennis serve performance. J Strength Cond Res 2004;18:654-6.

36. Gelen E, Dede M, Bingul BM, Bulgan C, Aydin M. Acute effects of static stretching, dynamic exercises, and high volume upper extremity plyometric activity on tennis serve performance. J Sports Sci Med 2012;11:600-5.

37. Cohen DB, Mont MA, Campbell KR, Vogelstein BN, Loewy JW. Upper extremity physical factors affecting tennis serve velocity. Am J Sports Med 1994;22:746-50.

38. Forthomme B, Croisier JL, Ciccarone G, Crielaard JM, Cloes M. Factors correlated with volleyball spike velocity. Am J Sports Med 2005;33:1513-9.

39. Bandy WD, Irion JM, Briggler M. The effect of time and frequency of static stretching on flexibility of the hamstring muscles. Phys Ther 1997;77:1090-6.

40. Magnusson P, Renstrom P. The European College of Sports Sciences Position statement: The role of stretching exercises in sports. European Journal of Sport Science 2006;6:87-91. 